\title{
Construction of a Crisp Boolean Petri Net from a 1-safe Petri Net
}

\author{
Gajendra Pratap Singh \\ Department of Applied Mathematics \\ Delhi Technological University \\ Shahbad Daulatpur, Main Bawana Road \\ Delhi-110042, India
}

\author{
Sangita Kansal \\ Department of Applied Mathematics \\ Delhi Technological University \\ Shahbad Daulatpur, Main Bawana Road \\ Delhi-110042, India
}

\author{
Mukti Acharya \\ Department of Applied Mathematics \\ Delhi Technological University \\ Shahbad Daulatpur, Main Bawana Road \\ Delhi-110042, India
}

\begin{abstract}
The concept of a Petri net, a tool for the study of certain discrete dynamical systems, was invented in 1939 by Carl Adam Petri. In the attempt to characterize Boolean Petri nets, we discovered a subclass of Boolean Petri net called the crisp Boolean Petri net, viz., the one that generates every binary vector as its marking vectors exactly once. In this paper, the construction of a crisp Boolean Petri net from a 1-safe Petri net has been shown.
\end{abstract}

\section{Keywords:}

1-safe Petri nets, graph theory, reachability tree, binary n-vector, hypercube.

\section{INTRODUCTION}

Petri nets are very reliable tool to model and study the structure of the discrete event-driven systems with large population or heavy traffic appear frequently in many fields such as manufacturing processes, logistics, telecommunication systems, traffic systems etc. The development of high-end computers has greatly enhanced the use of Petri nets in diverse fields. However, there is a drawback inherent to discrete event-system they suffer when there is no bound on the number of tokens on some place, the reachability graph may be infinite. This phenomenon leads to an exponential growth of the cardinality of the set of markings which, in turn, would blow up the 'size' (i.e., the number of arcs) of the system. This makes us to study the safe systems deeply. In [1], the authors proposed a 1-safe star Petri net $S_{n}$, with $|P|=n$ and $|T|=n+1$, having a central transition, that generates all the binary $n$-vectors, as its marking vectors. For brevity, a 1 -safe Petri net that generates all the binary $n$-vectors as marking vectors will be called a Boolean Petri net [2] and a 1-safe Petri net that generates all the binary $n$-vectors exactly once as marking vectors a crisp Boolean Petri net [2].

\section{PRELIMINARIES}

For standard terminology and notation on Petri net theory and graph theory, Readers are referred to Peterson [3] and Harary [4]. The following definition due to Jenson [5], has been adopted in this paper.

A Petri net is a 5-tuple $N=\left(P, T, I^{-}, I^{+}, \mu^{0}\right)$, where

(1) $P$ is a nonempty set of 'places',

(2) $T$ is a nonempty set of 'transitions',

(3) $P \cap T=\emptyset$,

(4) $I^{-}, I^{+}: P \times T \longrightarrow \mathbb{N}$, where $\mathbb{N}$ is the set of nonnegative integers, are called the negative and the positive 'incidence functions' (or, 'flow functions') respectively,

(5) $\forall p \in P, \exists t \in T: I^{-}(p, t) \neq 0$ or $I^{+}(p, t) \neq 0$ and $\forall t \in T, \exists p \in P: I^{-}(p, t) \neq 0$ or $I^{+}(p, t) \neq 0$,

(6) $\mu^{0}: P \rightarrow \mathbb{N}$ is the initial marking.

In fact, $I^{-}(p, t)$ and $I^{+}(p, t)$ represent the number of arcs from $p$ to $t$ and $t$ to $p$ respectively. $I^{-}, I^{+}$and $\mu^{0}$ can be viewed as matrices of size $|P| \times|T|,|P| \times|T|$ and $|P| \times 1$, respectively.

As in many standard books (e.g., see [6]), Petri net is a particular kind of directed graph, together with an initial marking $\mu^{0}$. The underlying graph of a Petri net is a directed, weighted, bipartite graph consisting of two kinds of nodes, called places and transitions, where arcs are either from a place to a transition or from a transition to a place. Hence, Petri nets have a well known graphical representation in which transitions are represented as boxes and places as circles with directed arcs interconnecting places and transitions to represent the flow relation. The initial marking is represented by placing a token in the circle representing a place $p_{i}$ as a black dot whenever $\mu^{0}\left(p_{i}\right)=1,1 \leq i \leq n=|P|$. In general, a marking $\mu$ is a mapping $\mu: P \longrightarrow \mathbb{N}$. A marking $\mu$ can hence be represented as a vector $\mu \in \mathbb{N}^{n}, n=|P|$, such that the $i^{t h}$ component of $\mu$ is the value $\mu\left(p_{i}\right)$.

Let $N=\left(P, T, I^{-}, I^{+}, \mu\right)$ be a Petri net. A transition $t \in T$ is said to be enabled at $\mu$ if and only if $I^{-}(p, t) \leq \mu(p), \forall p \in$ $P$. An enabled transition may or may not 'fire' (depending on whether or not the event actually takes place). After firing at $\mu$, the new marking $\mu^{\prime}$ is given by the rule

$$
\mu^{\prime}(p)=\mu(p)-I^{-}(p, t)+I^{+}(p, t), \forall p \in P .
$$


and write $\mu \stackrel{t}{\longrightarrow} \mu^{\prime}$, whence $\mu^{\prime}$ is said to be directly reachable from $\mu$. Hence, it is clear, what is meant by a sequence like

$$
\mu^{0} \stackrel{t_{1}}{\longrightarrow} \mu^{1} \stackrel{t_{2}}{\longrightarrow} \mu^{2} \stackrel{t_{3}}{\longrightarrow} \mu^{3} \cdots \stackrel{t_{k}}{\longrightarrow} \mu^{k},
$$

which simply represents the fact that the transitions $t_{1}, t_{2}, t_{3}, \ldots, t_{k}$ have been successively fired to transform the marking $\mu^{0}$ into the marking $\mu^{k}$. The whole of this sequence of transformations is also written in short as $\mu^{0} \stackrel{\sigma}{\longrightarrow} \mu^{k}$, where $\sigma=t_{1}, t_{2}, t_{3}, \ldots, t_{k}$ denotes the corresponding firing sequence.

A place in a Petri net is safe if the number of tokens in that place never exceeds one. A Petri net is safe if all its places are safe.

The preset of a transition $t$ is the set of all input places to $t$, i.e., ${ }^{\bullet} t=\left\{p \in P: I^{-}(p, t)>0\right\}$. The postset of $t$ is the set of all output places from $t$, i.e., $t^{\bullet}=\left\{p \in P: I^{+}(p, t)>0\right\}$. Similarly, $p^{\prime} s$ preset and postset are ${ }^{\bullet} p=\left\{t \in T: I^{+}(p, t)>0\right\}$ and $p^{\bullet}=\left\{t \in T: I^{-}(p, t)>0\right\}$, respectively.

Let $N=\left(P, T, I^{-}, I^{+}, \mu^{0}\right)$ be a Petri net with $|P|=n$ and $|T|=m$, the incidence matrix $I=\left[a_{i j}\right]$ is an $n \times m$ matrix of integers and its entries are given by $a_{i j}=a_{i j}^{+}-a_{i j}^{-}$, where $a_{i j}^{+}=I^{+}\left(p_{i}, t_{j}\right)$ is the number of arcs from transition $t_{j}$ to its output place $p_{i}$ and $a_{i j}^{-}=I^{-}\left(p_{i}, t_{j}\right)$ is the number of arcs from place $p_{i}$ to its output transition $t_{j}$, i.e., in other words, $I=I^{+}-I^{-}$.

\section{SOME DEFINITIONS}

DEFINITION 1. [7] A pair of a place $p$ and a transition $t$ is called a self-loop in a Petri net if $p$ is both an input and output place of $t$ (see. Figure 1).

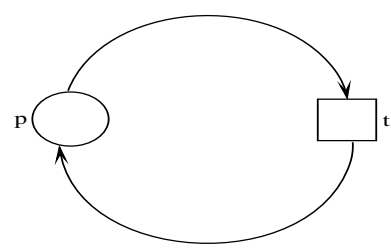

Fig. 1. Self-loop between a place $p$ and a transition $t$.

Definition 2. [2] Let $N=\left(P, T, I^{-}, I^{+}, \mu^{0}\right)$ be a Petri net and $Z$ be a subnet of $N$. Then $Z$ is called a strong chain cycle (SCC) of $N$ or $N$ is said to have a strong chain cycle (SCC) $Z$, if $|\bullet t|=2,\left|p^{\bullet}\right|=2$ and $\left|t^{\bullet}\right|=1 \forall p, t \in Z$. If an SCC $Z$ contains all the places of $N$ then $N$ is said to have a strong chain cycle covering all the places. Note that an SCC containing $k$ places, where $k \leq n=|P|$ will always have $k$ self-loops.

A Petri net with $n$ places and $n$ transitions having an SCC covering all the $n$ places is shown in Figure 2. A Petri net with 3 places

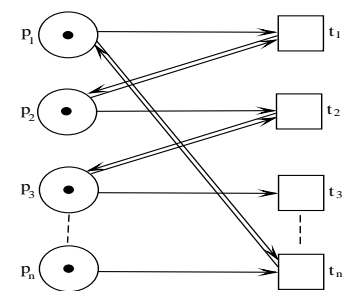

Fig. 2. Petri net having an SCC covering all the places and 3 transitions having an SCC not covering all the 3 places is shown in Figure 3.

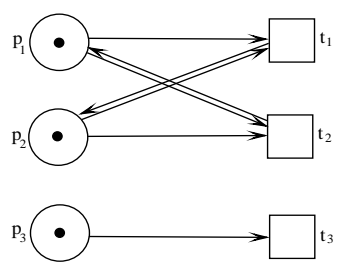

Fig. 3. Petri net having an SCC not covering all the places

DEFINITION 3. [8] $N_{n, n}^{*}$ is a Petri net with $n$ places and $n$ transitions having the following properties (i) The $i^{\text {th }}$ transition $t_{i}$ is contained in exactly $n-i$ self-loops, $i=1,2, \cdots, n$ (ii) The incidence matrix $I$ is the identity matrix $I_{n}$ of order $n$, and (iii) It does not contain

as a sub-Petri net.

For $n=3$ places, $N_{3,3}^{*}$ is shown in Figure 4 .

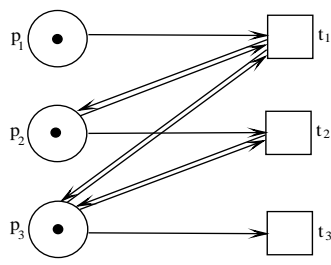

Fig. 4. $N_{3,3}^{*}$

Now, we first give some theorems which are needed for constructing a crisp Boolean Petri net.

THEOREM 4. [9] There exists a 1-safe Petri net with the initial marking $\mu^{0}(p)=1, \forall p \in P$ which generates each of the $2^{n}$ binary $n$-vectors

$$
\left(a_{1}, a_{2}, a_{3}, \cdots, a_{n}\right), a_{i} \in\{0,1\}, n=|P|,
$$

as one of its marking vectors, exactly once.

This Petri net has the structure as shown in Figure 5.

The total number of transitions $=2^{n}-1$

$\left|p^{\bullet}\right|=2^{n}-1, \forall p \in P$,

$|\cdot p|=2^{n-1}-1, \forall p \in P$,

$\bullet t \mid=n, \forall t \in T$.

Let ${ }^{n} C_{k}$ denote the combinatorial function that gives the number of distinct ways in which $k$ objects can be selected out of $n$ objects.

Then, the total number of transitions whose post-sets have $n-1$ elements $={ }^{n} C_{n-1}={ }^{n} C_{1}=n$ and these transitions are $t_{1}, t_{2}, t_{3}, \cdots, t_{n}$.

The total number of transitions whose post-sets have $n-2$ elements $={ }^{n} C_{n-2}={ }^{n} C_{2}=\frac{n(n-1)}{2}$ and these transitions are $t_{n+1}, t_{n+2}, t_{n+3}, \cdots, t_{\frac{n^{2}+n}{2}}$. 


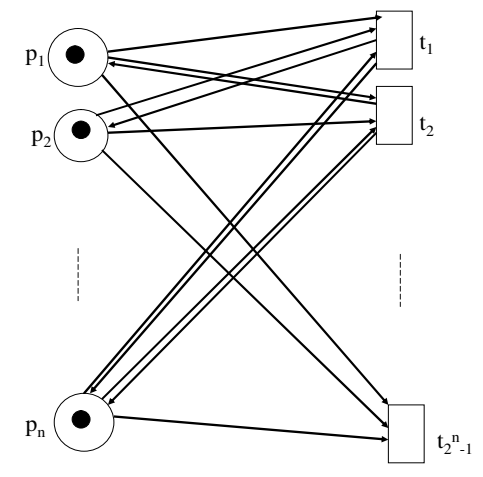

Figure 5: The minimal crisp Boolean Petri net.

The total number of transitions whose post-sets have $n-3$ elements $={ }^{n} C_{n-3}={ }^{n} C_{3}=\frac{n(n-1)(n-2)}{6}$ and these transitions are $t_{\frac{n^{2}+n+2}{2}}, t_{\frac{n^{2}+n+4}{2}}, t_{\frac{n^{2}+n+6}{2}}, \cdots, t_{\frac{n^{3}+5 n}{6}}$.

The total number of transitions whose post-sets have one element $={ }^{n} C_{1}=n$ and these transitions are $t_{2^{n}-n-1}, t_{2^{n}-n}, t_{2^{n}-n+1}, \cdots, t_{2^{n}-2}$.

The total number of transitions whose post-sets have no element $={ }^{n} C_{0}=1$ and this transition is $t_{2^{n}-1}$.

It has been proved in [10] that this Petri net is unique.

Theorem 5. [8] A 1-safe Petri net $N_{n, n}:=N_{n, n}^{*}$ with $\mu^{0}(p)=1, \forall p \in P$, is a minimum crisp Boolean Petri net.

\section{MAIN RESULT}

The two theorems mentioned above will be helpful in proving the following theorem which is the main result of this paper.

THEOREM 6. A crisp Boolean Petri net can be constructed from a 1-safe Petri net $N=\left(P, T, I^{-}, I^{+}, \mu^{0}\right)$.

Proof. Let $N=\left(P, T, I^{-}, I^{+}, \mu^{0}\right)$ be a 1 -safe Petri net. If $N$ is crisp Boolean then nothing to construct. Suppose $N$ is not crisp Boolean. Now two cases arise.

Case-1: $N$ contains a strong chain cycle (SCC).

Case-2: $N$ does not contain a strong chain cycle (SCC).

In case-1, when $N$ has an SCC, two subcases arise:

Subcase-1: If $|P|<|T|$ then increase the number of places or transitions or both (if necessary) such that the $|T|$ in the resulting Petri net $N^{\prime}$ becomes $2^{\text {number of places }}-1$. Now add the required arcs between places and transitions so that the structure of the new Petri net $N^{\prime}$ becomes the existing Petri net as given in the Theorem 1. By taking the initial marking vector $(1,1,1, \cdots, 1)$, one can see that $N^{\prime}$ is crisp Boolean Petri net. If in the given Petri net the $|T|$ is already $2^{|P|}-1$ then only by adding required arcs between places and transitions we get the new Petri net $N^{\prime}$, shown in Theorem 1 . Now by taking the initial marking vector $(1,1,1, \cdots, 1), N^{\prime}$ generates all the binary vectors exactly once.
Subcase-2: If $|P| \geq|T|$ then increase the number of transitions such that they would become $2^{|P|}-1$ in number. Then, by adding the required arcs between places and transitions we get the new Petri net which is shown in Theorem 1. By taking the initial marking vector $(1,1,1, \cdots, 1)$, one can see that $N^{\prime}$ is crisp Boolean Petri net.

Hence a crisp Boolean Petri net can be constructed from a 1-safe Petri net having an SCC.

In case-2, when $N$ does not contain an SCC, two subcases arise.

Subcase-1: If $|P|<|T|$ or $|P|>|T|$ then increase the $|T|-|P|$ number of places or $|P|-|T|$ number of transitions so that the resulting Petri net contains the equal number of places and transitions. Now add those arcs between places and transitions so that the structure of the new Petri net $N^{\prime}$ becomes the structure of $N_{n, n}^{*}$. By taking the initial marking vector $(1,1,1, \cdots, 1)$, $N^{\prime}$ generates all the binary vectors exactly once (By Theorem 2 ).

Subcase-2: If $|P|=|T|$ then only by adding the required arcs, the new Petri net $N^{\prime}$ becomes $N_{n, n}^{*}$. By taking the initial marking vector $(1,1,1, \cdots, 1)$, it is crisp Boolean Petri net. Therefore, a crisp Boolean Petri net cab be constructed from a 1-safe Petri net which does not contain an SCC.

Hence, theorem follows.

\section{CONCLUSIONS AND SCOPE}

This paper shows the construction of a crisp Boolean Petri net from a 1-safe Petri net. It is desirable to have a crisp Boolean Petri net because one may possibly explore for existence of certain sequences of enabled transitions to fire toward initiating and completing a prescribed process that uses specified nodes of the Boolean lattice. For example, in the design of generalized switches such as those used to control automatic machines [11], suppose that we have a sequence of $\mathrm{n}$ terminals each of which can be either at a prescribed low-voltage (denoted by zero ' 0 ') or at a prescribed high-voltage (denoted by unity, ' 1 '). It is required to arrange them so that every one of the $2^{n}$ sequences of $n$ bits, corresponding to the $2^{n}$ binary $n$-tuples, can appear on the terminals [11]. Due to the applicability of crisp Boolean Petri net into the diverse field, these have been a hotly pursued research problems. The result of this paper can perhaps be used gainfully in many purely theoretical areas like mathematics, computer science, universal algebra and order theory. Further, a Petri net containing an SCC is strongly connected, in the graph-theoretical sense that any two nodes in it are mutually reachable. However, the converse is not true; that is, if the underlying digraph of a Petri net is strongly connected, it need not contain an SCC. So, even a characterization of strongly connected Boolean Petri net is an open problem. Further, in general, characterizing crisp Boolean Petri nets is open too.

\section{REFERENCES}

[1] Kansal, S., Singh, G. P. and Acharya, M., On Petri nets generating all the binary $n$-vectors, Scientiae Mathematicae Japonicae, 71(2), 2010, 209-216, e-2013, 113-120.

[2] Kansal, S., Acharya, M. and Singh, G.P., Boolean Petri nets. In: Petri nets - Manufacturing and Computer Science (Ed.: Pawel Pawlewski), 381-406; Chapter 17. InTech Global Publisher, 2012, ISBN 978-953-51-0700-2.

[3] Peterson, J.L., Petri net theory and the modeling of systems, Englewood Cliffs, NJ: Prentice-Hall, Inc., 1981.

[4] Harary, F., Graph theory, Addison-Wesley, Massachusettes, Reading, 1969. 
[5] Jensen, K., Coloured Petri nets, Lecture notes in computer science, 254, Springer-Verlag, Berlin, 1986, 248299.

[6] Reisig, W., Petri nets, Springer-Verleg, New York, 1985.

[7] Murata, T., Petri nets: Properties, analysis and applications, Proc. IEEE, 77(4), 1989, 541-580.

[8] Singh, G.P., Some advances in the theory of Petri nets, Ph.D. Thesis, submitted to the University of Delhi, Delhi, December 5, 2012.

[9] Kansal, S., Singh, G.P. and Acharya, M., 1-Safe Petri nets generating every binary $n$-vector exactly once, Scientiae
Mathematicae Japonicae, 74(1), 2011, 29-36, e-2011, 127 134.

[10] Kansal, S., Acharya, M. and Singh, G.P., Uniqueness of minimal 1-safe Petri net generating every binary $n$-vectors as its marking vectors exactly once, Scientiae Mathematicae Japonicae, e-2012, 75-78.

[11] Acharya, B.D., Set-indexers of a graph and set-graceful graphs, Bull. Allahabad Math. Soc. 16, 2001, 1-23. 